\title{
Sodium-glucose cotransporter-2 inhibitors: current knowledge on the use of canagliflozin, dapagliflozin and empagliflozin in the treatment of type 2 diabetes mellitus
}

\author{
Malyn Martha Lilac Ketisha Antoine ${ }^{1}$, Yancheng $\mathrm{Xu}^{1 *}$ \\ ${ }^{1}$ Department of Endocrinology, Zhongnan Hospital of Wuhan University, Hubei China \\ *Corresponding author E-mail: xjl100901@whu.edu.cn
}

\begin{abstract}
Sodium-glucose cotransporter-2 inhibitors are considered the newest class of medication developed for the treatment of type 2 diabetes mellitus. This class of drug reduces blood glucose levels by decreasing glucose absorption at the level of the kidneys. Through their mechanism of action, drugs within this class exhibit the ability to reduce blood pressure, fasting blood glucose and body weight. The common side effects observed with the use of sodium-glucose cotransporter-2 inhibitors include: genital mycotic infections, intravascular volume depletion and dehydration. To date, much is already known about Sodium-glucose cotransporter-2 inhibitors and their use in treating type 2 diabetes mellitus; however, continuous studies are currently being undertaken to further investigate the cardiovascular benefits and side effect profile of this class of drug. This review discusses sodium glucose cotransporter- 2 inhibitors specifically focusing on what is currently known about the use of canagliflozin, dapagliflozin and empagliflozin in the treatment of type 2 diabetes mellitus.
\end{abstract}

Keywords: Canagliflozin; Dapagliflozin; Empagliflozin; Type 2 Diabetes Mellitus.

\section{Introduction}

According to the World Health Organization (WHO), in 2014, it was estimated that 422 million people were living with diabetes mellitus with greater prevalence of the condition being observed amongst developing countries ('Diabetes', n.d.). Information from the International Diabetes Federation (IDF), states that in 2011, approximately 366 million individuals suffered from diabetes mellitus and it is projected that by the year 2030, 552 million individuals will be affected by this chronic illness (Whiting et al. 2011) . In addition, in 2017 the IDF stated that approximately 451 million people worldwide between the ages of 18-99 years were living with diabetes. This source also reveals that during this period (2017) approximately 5 million deaths worldwide were secondary to diabetes (Cho et al. 2018). The upward trend observed in the prevalence of diabetes, has currently led to an urgent need for the further development of anti-diabetic medications that has the potential to effectively manage the condition along with its complications.

Investigations into the kidneys and their role in glucose homeostasis have led to the development of a new class of medication called sodium-glucose cotransporter-2 (SGLT2) inhibitors. This discovery has currently brought into sight a very promising view for the future management of type 2 diabetes mellitus (T2DM). Predominantly, through the utilization of sodium-glucose cotransporter-2 (SGLT2) proteins, the kidneys play an essential role in glucose regulation. Therefore, by selectively blocking SGLT2 proteins, SGLT2 inhibitors have been proven significant in decreasing blood glucose levels along with blood pressure and body weight (Wilding 2014, Bommel et al. 2017). To date many drugs from this class have received approval for use in both the United States and Japan (Nasiri-Ansari et al. 2018, Boeder \& Edelman 2019).

Cardiovascular diseases is one of the major complications that leads to death in type 2 diabetic patients; therefore, it is imperative that newly developed anti-diabetic drugs not only target glucose control but also target the complication of cardiovascular diseases. To date, research has contributed greatly to the knowledge of SGLT2 inhibitors especially regarding their cardio-protective benefits (Tanaka \& Node 2017). This sheds a promising light on the future management of T2DM and its complications. Therefore, this review paper will closely examine the current status of SGLT-2 inhibitors in the management of T2DM focusing particularly on empagliflozin, canagliflozin and dapagliflozin.

\section{Function of SGLT2 proteins in glucose absorption}

Several decades of studies into the concept of glucosuria, through the use of a compound called phlorizin (a non-specific sodium glucose co-transporter inhibitor), have led to the discovery of various types of SGLT proteins. SLGT proteins do not depend on insulin for their normal function and their selective inhibition demonstrates a favourable effect on carbohydrate metabolism. Currently, many studies are being conducted to establish the potential effects that the use of SGLT protein inhibitors would have on the future management of T2DM (Hsia et al 2017). 
Most of the glucose freely filtered by the renal glomerulus is reabsorbed at the level of the proximal renal tubules (Mosley et al. 2015). The reabsorption of glucose at this level of the nephron is an active process facilitated by sodium coupled transporters which are predominantly found on the apical surface (brush border) of the proximal tubular epithelial cells. Sodium glucose cotransporter-1(SGLT1) and sodium-glucose cotransporter-2 (SGLT2) proteins are the two major sodium coupled transport proteins found in the proximal tubules of the kidneys (Mather \& Pollock 2011).

Physiologically, the proximal renal tubule is divided into three segments: Segment1 (S1), Segment 2 (S2) and Segment 3 (S3). SGLT2 proteins are mainly found in Segments 1 and 2 of the proximal tubules. These proteins exhibit a low affinity for glucose transport; however, the majority of glucose filtered by the glomerulus (90\%) is reabsorbed by SGLT2 proteins due to the high capacity that these proteins exhibit for glucose transport (Gerich 2010). These receptors allow for the concomitant transportation of both sodium $\left(\mathrm{Na}^{+}\right)$and glucose. At the apical surface of the tubular epithelial cells, a Na+ gradient exist across the cells' membrane. This is important as it is required to provide the energy needed to transport glucose into the cell against its concentration gradient ${ }^{11}$. This Na+ gradient is maintained by sodiumpotassium-adenosine triphosphatase (Na+-K+ ATPase) pumps found at the basolateral aspect of the tubular epithelial cells. The movement of sodium along its electrochemical gradient produces energy which permits for one molecule of glucose to be concomitantly transported into the cell against its concentration gradient. This simultaneous movement of $\mathrm{Na}+$ and glucose are aided predominantly through the use of SGLT2 proteins (Jaikumkao et al. 2017).

\section{Gliflozins (SGLT2 inhibitors)}

As is already established, SGLT2 proteins reabsorb the majority of glucose filtered by the glomerulus and therefore, they play a major role in glucose homeostasis. As a result, great attention is now given to these proteins regarding their role in the management of type 2 diabetes mellitus. This has led to the development of different SGLT2 inhibitors all of which displays varying degrees of efficacy and selectivity (Isaji 2011).

\subsection{Canagliflozin}

From the list of currently available SGLT2 inhibitors, canagliflozin is the first drug in its class to receive approval for use in the United States of America (USA), receiving US FDA approval in March of 2013 (Plosker 2014). Canagliflozin is administered at a start dose of $100 \mathrm{mg}$ per day. In cases where tolerance develops and further glucose control is required this dose can be adjusted to $300 \mathrm{mg}$ in individuals with normal renal functions; however, patients with renal impairments should not receive a dose of more than 100mg of canagliflozin per day (Moses et al. 2014, Vivian 2014, Carbone \& Dixon 2019).

Canagliflozin exhibits a bioavailability of about $65 \%$. After oral administration of doses between 100mg-300mg, it takes approximately 12 hours for canagliflozin to reach peak blood levels and about 4-5 days to achieve steady state concentrations. At doses of $100 \mathrm{mg}$ and 300 mg daily, canagliflozin has an approximated half- life of 10.6 hours and 13.1 hours respectively (Kaushal et al. 2014, Carbone \& Dixon 2019). Once in the body, this drug is acted upon and broken down through a process called glucuronidation, converting the drug into its inactive form. A small percentage of canagliflozin (approximately 7\%) is also metabolized by oxidative (CYP3A4) metabolism (Plosker 2014).

Canagliflozin given at a dose ranging between $50 \mathrm{mg}-600 \mathrm{mg}$ per day has the ability to significantly reduce fasting blood glucose levels ( 0.9 to $2.1 \mathrm{mmol} / \mathrm{L}$ reduction). Canagliflozin has also been demonstrated to reduce HBA1c levels by approximately $0.9 \%$ at a daily dose of 300mg. In addition, due to the diuretic effect that this drug induces and the caloric loss that it promotes, canagliflozin has been shown to decrease blood pressure (systolic and diastolic) and body weight in patients with T2DM (Kaushal et al. 2014).

CANVAS (Canagliflozin Cardiovascular Assessment Study) study was carried out in type 2 diabetic (T2D) patients to investigate the safety profile of canagliflozin versus placebo in the presence of established cardiovascular treatment regimen. In this study canagliflozin was shown to contribute to a reduction in the frequency of deaths secondary to cardiovascular events. It was also proven effective in reducing blood pressure, HBA1c levels and body weight while increasing high density lipoprotein (HDL) levels (Skelley et al. 2018, Carbone \& Dixon 2019).

The use of canagliflozin is contraindicated in certain populations of patients. According to a phase 3 clinical trial, canagliflozin used in T2D patients experiencing stage 3 kidney diseases (CKD) yielded no major adverse effects; however, this study also showed that the drug is not suitable for patients experiencing severe kidney dysfunction (Madaan et al. 2016).

\subsection{Dapagliflozin}

The literature highlights that dapagliflozin is amongst the first SGLT2 inhibitors to receive worldwide approval for the treatment of T2DM. November 12, 2012 brought approval of this drug by the European Union. In July of 2011 alarming concerns regarding dapagliflozin's side effect profile (possible involvement in the development of liver injury and other conditions such as breast and bladder cancer) persuaded the Endocrinology and Metabolic Drugs Advisory Committee (EMDAC) of the FDA to issue warnings against the use of this drug. However, controversies surrounding this drug and its potential side effects were resolved and on January 8, 2014, dapagliflozin was accepted by the FDA for its use in the management of T2DM (Madaan et al. 2016).

The starting dose of dapagliflozin is recommended at $5 \mathrm{mg}$ which can be adjusted to $10 \mathrm{mg}$ if required. It is usually administered as a single dose and has an oral bioavailability of about $78 \%$ following a $10 \mathrm{mg}$ dosage. Following an oral dose of this drug it is rapidly absorbed achieving peak plasma levels within 30-120 minutes. At a dose of $10 \mathrm{mg}$, this drug has a half-life of approximately 13 hours. Dapagliflozin is majorly metabolized by an enzyme called uridine diphosphate-glucuronosyl-transferase (UGT) 1A9 to a compound called dapagliflozin 3-O- glucuronide; however, a small percentage of this drug can also be broken down by the CYP- metabolic pathway (Saeed \& Narendran 2014). This drug exists in the bloodstream mainly bound to plasma proteins; therefore, the percentage of the original drug excreted by the kidneys is discovered to be very low (<2\%) (Anderson, S.L., Marrs, J.C., n.d.).

Dapagliflozin induces a dose-dependent loss of glucose by the kidneys. A study conducted showed that T2DM patients who were administered $2.5-20 \mathrm{mg}$ of dapagliflozin, as a single dose, experienced a significant dose dependent increase in urinary excretion $(37.9-76.7 \mathrm{~g})$ of glucose as compared to the placebo group who only experienced a loss of urine glucose of about $9.4 \mathrm{~g}$ (Anderson, S.L., Marrs, J.C., n.d.). A meta-analysis was conducted to compare the effectiveness of SGLT2 inhibitors against established pharmacological therapies used in conjunction with other known glucose lower drugs. This study showed that dapagliflozin administered to study participants at a daily dose of $10 \mathrm{mg}$ for at least $12-26$ weeks contributed to a $0.54 \%(95 \% \mathrm{CI}-0.67 \%$ to $-0.40 \%$, p $<0.00001)$ reduction in HBA1c levels as compared 
to the placebo group. In addition, this study also demonstrated that dapagliflozin has the potential to promote weight reduction (Clar et al. 2012).

Research evidence suggests that dapagliflozin may have some cardiovascular benefits. A study was conducted to compare the use of dapagliflozin or placebo in T2D patients exhibiting increased risk for atherosclerotic cardiovascular disease. In this study, dapagliflozin contributed to a decrease in the frequency of hospitalizations secondary to heart failure. It was also observed that there was a reduction in the number of deaths that occurred secondary to cardiovascular events. In addition, this study demonstrated some cardio-protective benefits of dapagliflozin through an observed decrease in HBA1c levels (Wiviott et al. 2019).

The use of dapagliflozin may be contraindicated in patients with renal impairment. A randomized clinical trial implied that this drug produces no significant adverse effects in patients suffering from mild renal impairment. However, patients experiencing moderate renal dysfunction, displayed modest increase in renal associated adverse effects (Madaan et al. 2016). In the clinical setting, patients suffering from mild to moderate liver dysfunction do not require any adjustments in the administered dose of dapagliflozin. However, patients with severe liver dysfunction can be started on $5 \mathrm{mg}$ of dapagliflozin and this dose can be increased to $10 \mathrm{mg}$ if no adverse effects are observed (Saeed \& Narendran 2014).

\subsection{Empagliflozin}

Empagliflozin received US FDA approval in August 2014, being amongst the last of its class to be approved by this body for the treatment of T2DM. It is currently administered at a $10 \mathrm{mg}$ once daily dosage. This dose can be increased to $25 \mathrm{mg}$ per day as required and empagliflozin can be administered in the presence or the absence of a meal. In addition, oral administration requires approximately $1.5 \mathrm{~h}$ to attain maximum blood concentration and this drug has an estimated half-life between 10.3-18.8 hours. Furthermore, it exhibits a bioavailability of approximately $78 \%$ and is metabolized by two processes: glucuronidation and oxidation (Chawla \& Chaudhary 2019).

This drug has been proven to reduce blood glucose, blood pressure and blood volume levels. A meta-analysis was conducted to assess the efficacy of empagliflozin in comparison to a placebo group in patients diagnosed with T2DM. Empagliflozin was administered singly or in combination with other anti-diabetic medications. This study revealed that empagliflozin exhibited the ability to reduce blood HBA1c levels, both when administered alone or conjunctly with other anti-diabetic drugs. In addition, this meta-analysis also revealed that this drug also has the ability to support weight loss and reduce blood pressure in T2D patients (Zhang et al. 2018).

To date, evidence has demonstrated that empagliflozin may have some cardio-protective effects. EMPA-REG OUTCOME (Empagliflozin Cardiovascular Outcome Event Trial in Type 2 Diabetic Patients- Removing Excess Glucose) is a study that was conducted to compare the use of empagliflozin to the standard of care of treatment in T2DM patients who were at high risk for developing cardiovascular diseases. This study revealed a reduction in the frequency of cardiovascular events (nonfatal myocardial infarction, cardiovascular deaths) and neurovascular events (nonfatal strokes) in addition to a decrease in the number of hospitalizations observed due to heart failure (HF). These outcomes were positively observed in the experimental group as compared to the placebo group (Staels 2017, Eliasson et al. 2019).

The use of empagliflozin in patients with stage 2 and 3 renal disease yields no significant adverse effects; however, patients in stage 4 renal failure have been shown to experience increased episodes of hypoglycaemia with the use of this drug and therefore, empagliflozin is not recommended for use in patients with severe renal dysfunction (Barnett et al. 2014).

Table 1: Comparing Canagliflozin, Dapagliflozin and Empagliflozin

\begin{tabular}{lllll}
\hline Drug & Recommended daily Dosage & Bioavailability (\%) & Time to Peak Plasma Concentration in hours (hrs.) & Half-life in hours (hrs.) \\
\hline Canagliflozin & $100-300 \mathrm{mg}$ & $65 \%$ & $1-2$ & $10.6-13.1$ \\
Dapagliflozin & $10-25 \mathrm{mg}$ & $78 \%$ & 13 & 13 \\
Empagliflozin & $5-10 \mathrm{mg}$ & $78 \%$ & $1.33-3^{-}$ & $10.3-18.8$ \\
\hline
\end{tabular}

\subsection{Adverse effects}

The side effect profile observed with the use of SGLT2 inhibitors is mainly attributed to their mechanism of action(Whalen et al. 2015).Studies show that the most commonly seen adverse effect in patients using SGLT2 inhibitors is urogenital mycotic infections. In addition, a small proportion of studies also demonstrate that this class of medication result in urinary tract infections (UTI) (Kalra 2014, Lupsa \& Inzucchi 2018). A meta-analysis which examined the use of SGLT2 inhibitors in comparison to a placebo group or other classes of anti-diabetic drugs in type 2 diabetic patients, demonstrated that genital mycotic infections were increased by 5 times with the use of SGLT2 inhibitors. This study also showed a small increase in the occurrence of UTI's (Vasilakou et al. 2013). These side effects, however, are not considered severe and therefore, do not interrupt the use of the drugs.

SGLT2 inhibitors also induce dehydration and hypotension as a result of their diuretic effects; however, randomized control trials have indicated that these effects are only marginal (Jung et al. 2014, Kalra 2014). In general, the risk of dehydration and hypotension with the use of SGLT2 inhibitors is greater in older patients (>75 years), patients with glomerular filtration rates (GFR) of less than $60 \mathrm{~L} / \mathrm{min}^{\prime} / 1.73 \mathrm{~m}^{2}$ and patients treated with loop diuretics. When used as a single drug therapy, SGLT-2 inhibitors pose minimal risks for the development of hypoglycaemia. However, this risk is significantly increased when this class of medication is used conjunctly with other anti-diabetic drugs ( (Kalra 2014, Thynne \& Doogue 2014).

Some rare side effects have also been observed with the use of SGLT2 inhibitors. In 2015, two published cases demonstrated the development of acute pancreatitis with associated diabetic ketoacidosis (DKA) in patients receiving canagliflozin over a specified period of time (Hsia et al 2017). Clinical trials with dapagliflozin were associated with the development of bladder cancer; therefore, it is recommended that this drug be used cautiously in patients suffering from bladder cancer or patients previously diagnosed with the disease (Anderson, S.L., Marrs, J.C., n.d.) .Canagliflozin and canagliflozin/metformin preparations have been associated with an increased risk of bone fractures. Recent data demonstrated an increased in the occurrence of bone fractures in patient receiving canagliflozin as compared to the placebo treatment (Levine 2017).

\section{Discussion and future outlook}

To date, much information have already been accumulated regarding SGLT2 inhibitors and this has raised marked interest for the use of this class of medication as a potential alternative for the treatment of T2DM. In the presence of a myriad of options for the treatment of T2DM, the effective management of this condition still remains a significant challenge (van Baar et al. 2018). Through the combination of 
different drug therapies many physicians often struggle to achieve optimal blood glucose control and thus prevent the onset of micro and macro vascular complications associated with the disease. In addition, in some instances where established drug therapies may prove to be effective, adverse side effects such as weight gain and hypoglycaemia create unfavourable conditions for the continued use of these treatment regimens (Gomes et al. 2019).

SGLT2 inhibitors, however, are a promising group of medication for the effective management of T2DM and its complications with the absence of common side effects such as weight gain and hypoglycaemia. The mechanism of action of canagliflozin, dapagliflozin and empagliflozin are insulin independent, therefore, they serve a perfect alternative for the treatment of T2DM even in patients with advanced stages of the disease. In addition, they can be used in conjunction with insulin therapy and can be very effective in treating patients with insulin resistance (Cefalu \& Riddle 2015, Thomas \& Cherney 2018). These drugs serve a promising future for the treatment of type 2 diabetes mellitus through their potential abilities to decrease HBA1c levels, reduce blood pressure, induce weight loss and influence changes in the blood lipid profiles (Scheen 2015). According to Brown et.al, current SGLT2 inhibitors have already been proven to be effective in decreasing HBA1c levels through their abilities to affect blood glucose levels. In addition, this source also reveals that drugs in this class have also been shown to reduce blood pressure and body weight (Brown et al. 2019).

The abnormal metabolic parameters (high blood pressure, high blood glucose, abnormal lipid profile) that these SGLT2 inhibitors control are all major risk factors for the development of cardiovascular diseases. Therefore, in the future, further research may serve to demonstrate that the cardio-protective effects of SGLT2 inhibitors may arise partly due to their ability to regulate not only the blood HBA1C levels but to also reduce hypertension, support weight loss and alter plasma lipid profiles. Two major clinical trials CANVAS and EMPA-REG OUTCOME, which investigated the cardio-protective effects of canagliflozin and empagliflozin, have already demonstrated the effects of these two drugs in decreasing adverse cardiovascular events in patients with T2DM (Satoh, 2018). In addition, a meta-analysis, which integrated different clinical trials that focused on the efficacy and safety of SGLT2 inhibitors, showed that the use of SGLT2 inhibitors demonstrated a significant reduction in deaths due to cardiovascular causes. In addition, this study also showed a reduced frequency in the hospitalization of patients secondary to heart failure (Zelniker et al. 2019). Inadequate glucose control is a major contributory factor to the development of micro-vascular complications as seen in T2D patients. The ability of SGLT2 inhibitors to reduce HBA1c levels demonstrates their potential benefits in decreasing or delaying the onset of micro-vascular complications in type 2 diabetics (Plosker, 2014). Despite the common side effects observed with the use of this class of medication, SGLT2 inhibitors, appear to be quite safe and well tolerated. Therefore they serve as the perfect alternative in instances where other anti-diabetic medications are proven ineffective or when other classes of anti-diabetic drugs produce unwanted adverse effects.

\section{Conclusion}

Despite the major benefits that diabetic patients purpose to derive from the use of these SGLT2 inhibitors, close attention should be given to their side effect profile which is not yet fully established. Future research is required to conclusively determine the extent of the cardioprotective nature of SGLT2 inhibitors in patients suffering from T2DM and to fully determine the side effects profile of this class of medication, particularly focusing on their effects on the bone, liver and pancreas. Research also needs to be done to determine the benefits of SGLT2 inhibitors in combination with modifying factors such as life style changes and diet.

\section{References}

[1] Anderson, S.L., Marrs, J.C., (n.d.). Dapagliflozin for the Treatment of Type 2 Diabetes-Amie McCord Brooks, Stacey M Thacker, 2009.

[2] Barnett, A. H., Mithal, A., Manassie, J., Jones, R., Rattunde, H., Woerle, H. J., \& Broedl, U. C. (2014). Efficacy and safety of empagliflozin added to existing antidiabetes treatment in patients with type 2 diabetes and chronic kidney disease: A randomised, double-blind, placebo-controlled trial. The Lancet Diabetes \& Endocrinology, 2(5), 369-384. https://doi.org/10.1016/S2213-8587(13)70208-0.

[3] Boeder, S., \& Edelman, S. V. (2019). Sodium-glucose co-transporter inhibitors as adjunctive treatment to insulin in type 1 diabetes: A review of randomized controlled trials. Diabetes, Obesity \& Metabolism, 21 Suppl 2, 62-77. https://doi.org/10.1111/dom.13749.

[4] Bommel, E. J. M. van, Muskiet, M. H. A., Tonneijck, L., Kramer, M. H. H., Nieuwdorp, M., \& Raalte, D. H. van. (2017). SGLT2 Inhibition in the Diabetic Kidney-From Mechanisms to Clinical Outcome. Clinical Journal of the American Society of Nephrology, 12(4), 700-710. https://doi.org/10.2215/CJN.06080616.

[5] Brown, E., Rajeev, S. P., Cuthbertson, D. J., \& Wilding, J. P. H. (2019). A review of the mechanism of action, metabolic profile and haemodynamic effects of sodium-glucose co-transporter-2 inhibitors. Diabetes, Obesity \& Metabolism, 21 Suppl 2, 9-18. https://doi.org/10.1111/dom.13650.

[6] Carbone, S., \& Dixon, D. L. (2019). The CANVAS Program: Implications of canagliflozin on reducing cardiovascular risk in patients with type 2 diabetes mellitus. Cardiovascular Diabetology, 18(1), 64. https://doi.org/10.1186/s12933-019-0869-2.

[7] Cefalu, W. T., \& Riddle, M. C. (2015). SGLT2 Inhibitors: The Latest "New Kids on the Block"! Diabetes Care, 38(3), 352-354. https://doi.org/10.2337/dc14-3048.

[8] Chawla, G., \& Chaudhary, K. K. (2019). A complete review of empagliflozin: Most specific and potent SGLT2 inhibitor used for the treatment of type 2 diabetes mellitus. Diabetes \& Metabolic Syndrome, 13(3), 2001-2008. https://doi.org/10.1016/j.dsx.2019.04.035.

[9] Cho, N. H., Shaw, J. E., Karuranga, S., Huang, Y., da Rocha Fernandes, J. D., Ohlrogge, A. W., \& Malanda, B. (2018). IDF Diabetes Atlas: Global estimates of diabetes prevalence for 2017 and projections for 2045. Diabetes Research and Clinical Practice, 138, 271-281. https://doi.org/10.1016/j.diabres.2018.02.023.

[10] Clar, C., Gill, J. A., Court, R., \& Waugh, N. (2012). Systematic review of SGLT2 receptor inhibitors in dual or triple therapy in type 2 diabetes. BMJ Open, 2(5), e001007. https://doi.org/10.1136/bmjopen-2012-001007.

[11] Diabetes. (n.d.). Retrieved 2 October 2019, from https://www.who.int/news-room/fact-sheets/detail/diabetes.

[12] Eliasson, B., Ekelund, J., Amberntsson, R., Miftaraj, M., \& Svensson, A.-M. (2019). Cardiovascular Disease in Patients with Type 2 Diabetes and in Patients Starting Empagliflozin Treatment: Nationwide Survey. Diabetes Therapy, 10(4), 1523-1530. https://doi.org/10.1007/s13300-019-0632-4.

[13] Gerich, J. E. (2010). Role of the kidney in normal glucose homeostasis and in the hyperglycaemia of diabetes mellitus: Therapeutic implications. Diabetic Medicine: A Journal of the British Diabetic Association, 27(2), 136-142. https://doi.org/10.1111/j.1464-5491.2009.02894.x.

[14] Gomes, M. B., Rathmann, W., Charbonnel, B., Khunti, K., Kosiborod, M., Nicolucci, A., DISCOVER investigators. (2019). Treatment of type 2 diabetes mellitus worldwide: Baseline patient characteristics in the global DISCOVER study. Diabetes Research and Clinical Practice, 151, 20-32. https://doi.org/10.1016/j.diabres.2019.03.024.

[15] Hsia, D. S., Grove, O., \& Cefalu, W. T. (2017). An Update on SGLT2 Inhibitors for the Treatment of Diabetes Mellitus. Current Opinion in Endocrinology, Diabetes, and Obesity, 24(1), 73-79. https://doi.org/10.1097/MED.0000000000000311.

[16] Isaji, M. (2011). SGLT2 inhibitors: Molecular design and potential differences in effect. Kidney International, 79, S14-S19. https://doi.org/10.1038/ki.2010.511. 
[17] Jaikumkao, K., Pongchaidecha, A., Chatsudthipong, V., Chattipakorn, S. C., Chattipakorn, N., \& Lungkaphin, A. (2017). The roles of sodium-glucose cotransporter 2 inhibitors in preventing kidney injury in diabetes. Biomedicine \& Pharmacotherapy = Biomedecine \& Pharmacotherapie, 94, 176187. https://doi.org/10.1016/j.biopha.2017.07.095.

[18] Jung, C. H., Jang, J. E., \& Park, J.-Y. (2014). A Novel Therapeutic Agent for Type 2 Diabetes Mellitus: SGLT2 Inhibitor. Diabetes \& Metabolism Journal, 38(4), 261-273. https://doi.org/10.4093/dmj.2014.38.4.261.

[19] Kalra, S. (2014). Sodium Glucose Co-Transporter-2 (SGLT2) Inhibitors: A Review of Their Basic and Clinical Pharmacology. Diabetes Therapy, 5(2), 355-366. https://doi.org/10.1007/s13300-014-0089-4.

[20] Kaushal, S., Singh, H., Thangaraju, P., \& Singh, J. (2014). Canagliflozin: A Novel SGLT2 Inhibitor for Type 2 Diabetes Mellitus. North American Journal of Medical Sciences, 6(3), 107-113. https://doi.org/10.4103/1947-2714.128471.

[21] Levine, M. J. (2017). Empagliflozin for Type 2 Diabetes Mellitus: An Overview of Phase 3 Clinical Trials. Current Diabetes Reviews, 13(4), 405423. https://doi.org/10.2174/1573399812666160613113556.

[22] Lupsa, B. C., \& Inzucchi, S. E. (2018). Use of SGLT2 inhibitors in type 2 diabetes: Weighing the risks and benefits. Diabetologia, 61(10), 21182125. https://doi.org/10.1007/s00125-018-4663-6.

[23] Madaan, T., Akhtar, Mohd., \& Najmi, A. K. (2016). Sodium glucose CoTransporter 2 (SGLT2) inhibitors: Current status and future perspective. European Journal of Pharmaceutical Sciences, 93, 244-252. https://doi.org/10.1016/j.ejps.2016.08.025.

[24] Mather, A., \& Pollock, C. (2011). Glucose handling by the kidney. Kidney International, 79, S1-S6. https://doi.org/10.1038/ki.2010.509.

[25] Moses, R. G., Colagiuri, S., \& Pollock, C. (2014). SGLT2 inhibitors: New medicines for addressing unmet needs in type 2 diabetes. The Australasian Medical Journal, 7(10), 405-415. https://doi.org/10.4066/AMJ.2014.2181.

[26] Mosley, J. F., Smith, L., Everton, E., \& Fellner, C. (2015). Sodium-Glucose Linked Transporter 2 (SGLT2) Inhibitors in the Management Of Type2 Diabetes: A Drug Class Overview. Pharmacy and Therapeutics, 40(7), 451-462.

[27] Nasiri-Ansari, N., Dimitriadis, G. K., Agrogiannis, G., Perrea, D., Kostakis, I. D., Kaltsas, G., ... Kassi, E. (2018). Canagliflozin attenuates the progression of atherosclerosis and inflammation process in APOE knockout mice. Cardiovascular Diabetology, 17(1), 106. https://doi.org/10.1186/s12933-018-0749-1.

[28] Plosker, G. L. (2014). Canagliflozin: A Review of Its Use in Patients with Type 2 Diabetes Mellitus. Drugs, 74(7), 807-824. https://doi.org/10.1007/s40265-014-0225-5.

[29] Saeed, M. A., \& Narendran, P. (2014). Dapagliflozin for the treatment of type 2 diabetes: A review of the literature. Drug Design, Development and Therapy, 8, 2493-2505. https://doi.org/10.2147/DDDT.S50963.

[30] Satoh, H. (2018). Pleiotropic effects of SGLT2 inhibitors beyond the effect on glycemic control. Diabetology International, 9(4), $212-214$. https://doi.org/10.1007/s13340-018-0367-x.

[31] Scheen, A. J. (2015). Pharmacodynamics, Efficacy and Safety of Sodium-Glucose Co-Transporter Type 2 (SGLT2) Inhibitors for the Treatment of Type 2 Diabetes Mellitus. Drugs, 75(1), 33-59. https://doi.org/10.1007/s40265-014-0337-y.

[32] Skelley, J. W., Carter, B. S., \& Roberts, M. Z. (2018). Clinical potential of canagliflozin in cardiovascular risk reduction in patients with type 2 diabetes. Vascular Health and Risk Management, 14, 419-428. https://doi.org/10.2147/VHRM.S168472.

[33] Staels, B. (2017). Cardiovascular Protection by Sodium Glucose Cotransporter 2 Inhibitors: Potential Mechanisms. American Journal of Cardiology, 120(1), S28-S36. https://doi.org/10.1016/j.amjcard.2017.05.013.

[34] Tanaka, A., \& Node, K. (2017). Emerging roles of sodium-glucose cotransporter 2 inhibitors in cardiology. Journal of Cardiology, 69(3), 501-507. https://doi.org/10.1016/j.jjcc.2016.10.019.

[35] Thomas, M. C., \& Cherney, D. Z. I. (2018). The actions of SGLT2 inhibitors on metabolism, renal function and blood pressure. Diabetologia, 61(10), 2098-2107. https://doi.org/10.1007/s00125-018-4669-0.

[36] Thynne, T., \& Doogue, M. (2014). Sodium-glucose co-transporter inhibitors. Aust Prescr, 37, 14-6. https://doi.org/10.18773/austprescr.2014.005.

[37] Van Baar, M. J. B., van Ruiten, C. C., Muskiet, M. H. A., van Bloemendaal, L., IJzerman, R. G., \& van Raalte, D. H. (2018). SGLT2 Inhibitors in Combination Therapy: From Mechanisms to Clinical Considerations in Type 2 Diabetes Management. Diabetes Care, 41(8), 1543-1556. https://doi.org/10.2337/dc18-0588.

[38] Vasilakou, D., Karagiannis, T., Athanasiadou, E., Mainou, M., Liakos, A., Bekiari, E., ... Tsapas, A. (2013). Sodium-glucose cotransporter 2 inhibitors for type 2 diabetes: A systematic review and meta-analysis. Annals of Internal Medicine, 159(4), 262-274. https://doi.org/10.7326/0003-4819159-4-201308200-00007.

[39] Vivian, E. M. (2014). Sodium-glucose co-transporter 2 (SGLT2) inhibitors: A growing class of antidiabetic agents. Drugs in Context, 3. https://doi.org/10.7573/dic.212264.

[40] Whalen, K., Miller, S., \& Onge, E. S. (2015). The Role of Sodium-Glucose Co-Transporter 2 Inhibitors in the Treatment of Type 2 Diabetes. Clinical Therapeutics, 37(6), 1150-1166. https://doi.org/10.1016/j.clinthera.2015.03.004.

[41] Whiting, D. R., Guariguata, L., Weil, C., \& Shaw, J. (2011). IDF diabetes atlas: Global estimates of the prevalence of diabetes for 2011 and 2030. Diabetes Research and Clinical Practice, 94(3), 311-321. https://doi.org/10.1016/j.diabres.2011.10.029.

[42] Wilding, J. P. H. (2014). The role of the kidneys in glucose homeostasis in type 2 diabetes: Clinical implications and therapeutic significance through sodium glucose co-transporter 2 inhibitors. Metabolism, 63(10), 1228-1237. https://doi.org/10.1016/j.metabol.2014.06.018.

[43] Wiviott, S. D., Raz, I., Bonaca, M. P., Mosenzon, O., Kato, E. T., Cahn, A., ... DECLARE-TIMI 58 Investigators. (2019). Dapagliflozin and Cardiovascular Outcomes in Type 2 Diabetes. The New England Journal of Medicine, 380(4), 347-357. https://doi.org/10.1056/NEJMoa1812389.

[44] Zelniker, T. A., Wiviott, S. D., Raz, I., Im, K., Goodrich, E. L., Bonaca, M. P., ... Sabatine, M. S. (2019). SGLT2 inhibitors for primary and secondary prevention of cardiovascular and renal outcomes in type 2 diabetes: A systematic review and meta-analysis of cardiovascular outcome trials. The Lancet, 393(10166), 31-39. https://doi.org/10.1016/S0140-6736(18)32590-X.

[45] Zhang, Y.-J., Han, S.-L., Sun, X.-F., Wang, S.-X., Wang, H.-Y., Liu, X., ... Xia, L. (2018). Efficacy and safety of empagliflozin for type 2 diabetes mellitus. Medicine, 97(43). https://doi.org/10.1097/MD.0000000000012843. 